\title{
BRAIN INJURY FOLLOWING WHOLE BODY COOLING AFTER NEONATAL ENCEPHALOPATHY IN A SOUTH INDIAN NEONATAL UNIT
}

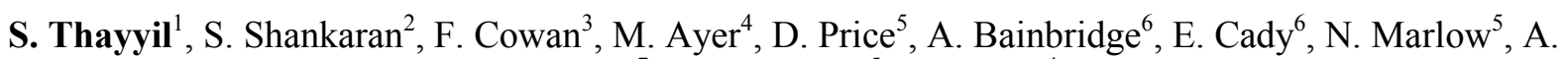

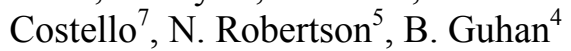 \\ ${ }^{I}$ Academic Neonatology, University College London, London, UK, ${ }^{2}$ Neonatal Perinatal Medicine, Wayne \\ State University Hospital, Detroit, MI, USA, ${ }^{3}$ Neonatal Medicine, Imperial College, London, UK, ${ }^{4}$ Neonatal \\ Medicine, Calicut Medical College, Calicut, India, ${ }^{5}$ Academic Neonatology, ${ }^{6}$ Medical Physics, ${ }^{7}$ Centre for \\ International Child Health, UCL, London, UK
}

Efficacy of therapeutic hypothermia (TH) in encephalopathic infants with co-existent sepsis is unknown; we assessed brain tissue injury using magnetic resonance (MR) biomarkers in a South Indian public sector neonatal unit.

Methods: We randomly allocated 33 infants (age $\geq 36$ weeks, birth weight $\geq 1800 \mathrm{~g}$ ) with neonatal encephalopathy (NE) (19 stage I, 11 stage II, 4 stage III) to TH (rectal temperature $33.5^{\circ} \mathrm{C}$ for 72 hours) or standard care, within 6 hours of birth. MR images were acquired between days 7 and 11 using a 1.5 Tesla Siemens Avanto and scored by a single examiner (FC).

Results: Baseline characteristics were similar in the cooled $(n=17)$ and standard care $(n=16)$ groups; 4 cooled and 2 standard care infants died. Trends towards increased sepsis (53\% versus $19 \%)$ and higher background EEG abnormality on day $4(47 \%$ versus $29 \%)$ were seen in the cooled infants. The MR imaging brain injury scores in the basal ganglia (odds ratio $(95 \% \mathrm{CI}) 3.4(0.5,22))$, white matter $(\mathrm{WM})(0.5(0.08,2.6)$ and cortex $(4.7(0.7,30)$ were similar in both groups. No intergroup differences were seen in tract based spatial statistics or in fractional anisotropy over the anterior and posterior limbs of the internal capsule.

Conclusions: TH did not reduce the brain injury apparent on MR imaging. This effect may be 'real' due to the different population co-morbidities in a high-sepsis setting or 'false' due to poor discriminatory power of the MR imaging biomarkers used ${ }^{2}$. Carefully controlled local clinical trials are required before TH is routinely in these settings. 